\title{
Culturally Informed Notions of Mobile Context Awareness Lessons Learned from User-centred Exploration of Concepts of Context and Context Awareness
}

\author{
Qin, Xiangang; Tan, Chee-Wee; Bødker, Mads; Sun, Wei; Clemmensen, Torkil
}

Document Version

Accepted author manuscript

Published in:

Human-Computer Interaction - INTERACT 2017

DOI:

10.1007/978-3-319-67744-6_27

Publication date:

2017

License

Unspecified

Citation for published version (APA):

Qin, X., Tan, C-W., Bødker, M., Sun, W., \& Clemmensen, T. (2017). Culturally Informed Notions of Mobile Context Awareness: Lessons Learned from User-centred Exploration of Concepts of Context and Context Awareness. In R. Bernhaupt, G. Dalvi, A. Joshi, D. K. Balkrishan, J. O’Neill, \& M. Winckler (Eds.), HumanComputer Interaction - INTERACT 2017: Proceedings of the 16th IFIP TC 13 International Conference. Part I (Vol. 10513, pp. 420-440). Springer. Lecture Notes in Computer Science Vol. 10513 https://doi.org/10.1007/9783-319-67744-6_27

Link to publication in CBS Research Portal

\section{General rights}

Copyright and moral rights for the publications made accessible in the public portal are retained by the authors and/or other copyright owners and it is a condition of accessing publications that users recognise and abide by the legal requirements associated with these rights.

\section{Take down policy}

If you believe that this document breaches copyright please contact us (research.lib@cbs.dk) providing details, and we will remove access to the work immediately and investigate your claim.

Download date: 26. Apr. 2023
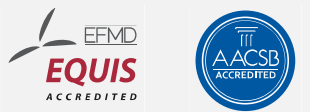


\title{
Culturally Informed Notions of Mobile Context Awareness: Lessons Learned from User-centred Exploration of Concepts of Context and Context Awareness
}

\author{
Kiangang Din, Chee-Wee Tan, Mads Bodker, Wei Sun, and Torkil Clemmensen \\ Journal article (Accepted version)
}

CITE: Culturally Informed Notions of Mobile Context Awareness : Lessons Learned from User-centred Exploration of Concepts of Context and Context Awareness. / Din, Xiangang; Tan, Chee-Wee; Bødker, Mads; Sun, Wei; Clemmensen, Torkil. In: Human-Computer Interaction - INTERACT 2017: Proceedings of the 16th IFIP

TC 13 International Conference. Part I. ed. / Regina Bernhaupt; Girish Dalvi; Anirudha Joshi; Devanuj K. Balkrishan; Jacki O'Neill; Marco Winckler. Cham : Springer, 2017. p. 420-440.

This is a post-peer-review, pre-copyedit version of an article published in Human-Computer Interaction - INTERACT 2017: Proceedings of the Ibth IFIP TC I3 International Conference. Part I

The final authenticated version is available online at: https://doi.org/10.1007/978-3-319-67744-6_27

Uploaded to CBS Research Portal: January २०19 


\title{
Culturally Informed Notions of Mobile Context Aware- ness - Lessons Learned from User-Centred Exploration of Concepts of Context and Context Awareness
}

\author{
Xiangang Qin ${ }^{1,2}$, Chee-Wee Tan ${ }^{1}$, Mads Bødker ${ }^{1}$, Wei Sun ${ }^{2}$, and Torkil Clemmensen ${ }^{1}$ \\ ${ }^{1}$ Copenhagen Business School, Frederiksberg, Denmark \\ \{xq.itm, cta.itm, mb.itm, tc.itm\}@cbs.dk \\ ${ }^{2}$ Beijing University of Posts \& Telecommunications, Beijing, China \\ \{qinxiangang, sunwey\} @bupt. edu.cn
}

\begin{abstract}
Mobile context awareness is an elusive concept within extant literature on human-computer interaction. Despite extensive research into context awareness, past studies have accentuated the architectural aspects of context-aware applications without paying sufficient attention to user-centric issues associated with such applications. To this end, this study endeavours to shed light on how context-aware applications are construed by users. Drawing inspiration from the 'future workshop' methodological approach, two focus groups were conducted in Denmark and China to elicit insights into the benefits and shortcomings of contemporary context-aware applications across different cultural settings. Empirical findings suggest that users' interactions with context-aware applications are governed, to a large extent, by their: (1) own personal value system; (2) sensitivity towards such applications, and; (3) current activity in which they are engaged. This study thus takes a small but concrete step towards further discussions on the importance of embracing a user-centred view of mobile context awareness.
\end{abstract}

Keywords: mobile context-awareness, user experience, cross-cultural

\section{Introduction}

The global population of smart phone users is projected to reach 2.32 billion by the end of 2017 [1]. Because a variety of sensors are embedded in smart phones which endow the latter with the ability to be aware of and interact with the surrounding context, smart phones can be conceived as a type of mobile context-aware system that extends human cognition and perception. Indeed, Mobile Context-Awareness (MCA) is a rapidly growing topic of interest for both academics and practitioners due to the increasing dynamism and richness of contextual information afforded by smart devices [2]. A variety of context-awareness systems have been developed to adapt system's behaviour with changing location, time, physical environment, identity and emotion state of user, nearby devices and social entities as well [3]. Although context-awareness is intended to circumvent issues in user experience (UX) caused by small screen size and evershifting context in smart devices, context-aware mobile applications are also presumed 
to be capable of offering more customized and more favourable UXs than non-contextaware ones [3, 5].It is accompanied by its own side effects such as distracting interruptions, loss of control and privacy risk [6]. There are calls for an in-depth appreciation of how the UX is shaped by mobile context-aware systems [5].

Yet, despite the calls, the UX of MCA and other user related issues have been lacking in HCI research. Hong et al [7] discovered that the bulk of research (237 articles in total) tends to concentrate on issues associated with conceptualization, network, middleware and application. Even though the few studies, which have examined the issue of UX for MCA (device and application), have put forth general guidelines for designing mobile context-aware systems (i.e., avoiding unnecessary interruptions, ensuring user control, guaranteeing system visibility and tailoring content to match individual needs) $[6,8]$, there is a dearth of research that has been devoted to a dedicated scrutiny of how such systems can be designed from a user-centred perspective in distinct cultural contexts. In addition, there is growing recognition that we have a limited comprehension of how to capture, interpret and share abstract contexts [9]. Social context is an exemplary illustration of an abstract context in that the UX of mobile context-aware system might vary in different social settings. We argue that culture plays an instrumental role in shaping UX of MCA, and should not be divorced from any investigative efforts targeted at the phenomenon. This study endeavours to unpack the concept of MCA from a user-centred perspective by eliciting user's opinion and experience with mobile context-aware systems in both Chinese and Danish cultural settings.

\section{Background}

\subsection{The Cultural Context of Mobile Services}

While emerging markets like China and India have already overtaken more matured markets like the United States in terms of mobile phone penetration, the UX of the popular smart devices (based on Android, iOS or Windows architecture) are still designed by Western firms. For this reason, Chinese firms are acquiring their Western counterparts in the provision of mobile services. However, consumers of both Chinese and Western firms are utilizing mobile services provided by companies with different cultural backgrounds such as Google vs. Baidu, Facebook vs. WeChat, Twitter vs. Weibo, to name a few. Users have developed corresponding habits of interacting with mobile applications that are designed and developed for different cultural contexts. It is unclear whether the design of mobile context-aware features by Chinese firms are accommodative of Westerners and vice versa.

The cultural context and user's preference of interaction might vary in different countries. Extant literature contains extensive evidence attesting to discrepancies between Asian and Western ways of acting and thinking [10]. Chinese subjects are more sensitive to contextual incongruity as compared to Americans [11]. Situation (or social context) influence the effect of culture on affect, cognition and behaviour. For this reason, intra- and inter-cultural variations of MCA can be best understood by taking the cultural context into consideration [12]. Specifically, Westerners are believed to be 
more concerned about the instrumental (efficiency) attributes of UX whereas Chinese users care more about non-instrumental (e.g., appearance) attributes of interactive products [13]. While a key merit of MCA resides in its ability to improve the efficiency of interactions, we contend that variations in users' attitudes toward MCA exist between Chinese and Denmark users. Despite a broad recognition of the presence of cross-cultural disparities in UX of mobile context-aware systems, e.g., [13], few studies investigate how and why such disparities exist, which in turn acts as the impetus for this study.

\subsection{The Value of Social Context and Context awareness}

Since the term 'context-aware' was first coined to describe the computing ability "of a mobile user's applications to discover and react to changes in the environment they are situated in" [14], context-awareness has been touted as a key component of pervasive and ubiquitous computing. Essentially, context-awareness endows mobile computing devices with the capability of interacting with humans in an intuitive manner that mirrors human-human interaction [15]. Nevertheless, it is only with the proliferation of smart devices that the importance of context awareness begins to gain traction.

For users, high-level contexts like activity, psychological status and place are more meaningful than low level contexts like location, noise, gravity, time. As a variable of high-level contexts, social context is valuable in deciphering and interpreting the intention and activities of human being by integrating and combing low-level context information captured by sensors. For example, providing contextual information about air quality would be valued more in Beijing than in Copenhagen. Yet, the social context is complicated and fast evolving in mobile situations, especially across different cultural contexts. A deeper appreciation of the focal dimensions of social context, which might influence the intentions and activities of users [5], would contribute to the abstraction, acquisition, inference and utilization of contextual information to enhance the UX of context-aware systems.

\subsection{The Influence of MCA on $\mathrm{HCl}$}

An abundance of intricate contextual information are exploited in human-to-human communication, such as eye contact, facial expression, hand gestures, body language and even more profound social attributes like culture and religions. Unlike human-tohuman communication, tradition interactions between humans and computers are constrained by the latter's computing power and number of embedded sensors in harvesting and harnessing contextual information [16, 17].

Over the past decade, mobile devices, especially smart phones, have been adopted by a vast population of users globally. In many countries, more than 50 percent of the population are smart phone users. Nowadays, mobile phones have been fitted with enhanced computing capabilities and miniaturized sensors that enable such phones to proactively interact with humans by drawing on contextual information to adapt to user intention and behaviours. Due to the infusion of context-aware features, smart devices are capable of interacting with users in intelligent, natural and human-like ways. 
Fundamentally, MCA alters the interaction between humans and computers in four ways. First, the interaction between users and context-aware systems is shifting from an explicit format to an implicit one. Second, the informational resources accessible to context-aware systems are much more diverse due to data generated by both humans and technological sensors [17]. Third, context-aware systems (e.g., smart phones) are transforming from a passive role of accepting, processing and displaying information to a more active role of abstracting, acquiring, inferring and utilizing contextual information in order to execute actions automatically. Finally, context-aware systems can sense other users and computing devices in their surroundings, thereby rendering crossdevice social interaction plausible.

\subsection{MCA from a User-Centred Perspective}

Designers, developers and users are inclined to interpret the same system from diverse perspectives, thereby culminating in separate meanings. One of the most valuable contribution of HCI to mobile system is that it bridges the gap among the mental models of the abovementioned three parties [18]. An early study on the usability of mobile context-aware applications demonstrated that while users prefer to utilize contextaware applications with higher degrees of proactivity, their feelings of out of control, lack of feedback, privacy leak and information overload increases correspondingly [8]. At the same time, users express a greater willingness to tolerate the preceding uncertainties in exchange for a more interactive and smoother experience. Because users are adaptive and context-dependent when utilizing context-aware mobile applications, it implies that the UX of same mobile application may vary across contexts and usage.

In mobile environments, the use context tends to fluctuate and the same contextual information might acquire differentiated meanings across distinct contexts and usage [19]. Since user profiles and purpose is likely to differ when utilizing mobile applications [3], it is conceivable for users to utilize the same application in contexts that were not anticipated by designers and developers [5]. Indeed, it is not uncommon for contextaware systems to encounter difficulties in interpreting user context due to variations in physical attributes (i.e., height, palm size and eye-sight), cognitive and perceptual abilities (e.g. memory, learning, problem-solving and decision making) and personality (e.g. gender, attitudes towards computers and emotional states).

Compared with the era dominated by desktop computers and feature phones, contexts associated with the utilization of smart mobile devices have changed dramatically and enriched significantly. Mobile applications tend to become much more complex as the quantity and types of information employed by context-aware applications have surpassed those of non-context-aware ones [6]. Mobile applications developed by Chinese company include more functionalities than those introduced by Western companies (e.g., wallet payment, red packet and games in WeChat), which in turn might bring about unexpected interactive behaviours and experiences.

To-date, context-awareness has mainly been investigated from a technical point of view with strong emphasis on location-aware mobile applications [5, 7]. The limited research about mobile context-aware system, from a user-centred perspective, are either ecologically invalid experiment, or too specific for guiding the design of applications 
in diverse fields [20]. Particularly, a major research trend within extant literature on mobile context-awareness systems is rooted in the design of middleware and APIs to facilitate the development of MCA applications. In the absence of an in-depth appreciation of users, contemporary studies run the risk that findings derived from inquiries of user-centred MCA applications may not be generalizable to other fields.

A number of questions emerge with respect to the impact of MCA on Human-Computer Interaction (HCI): How can users be aware of the intricate contextual information being inputted and captured by mobile context-aware systems? What's the concerns of users about mobile context-aware applications and how the concerns might shape the interaction between user and mobile device? How to exploit and integrate contextual information in multi-modals? Should smart phones be more active in executing actions that are undertaken by humans traditionally? How should humans deal with an intelligent and emotional device that has been endowed with social-networking features?

\section{Methods}

We opted for the Focus Group (FG) and Future Workshop(FW) methodology in this study because they has been acknowledged as an appropriate technique for conducting exploratory research on social phenomena and participatory design studies [e.g., 18, 22, $23,24,25]$. The future workshop approach departs from the standard FG format by placing greater emphasis on mapping techniques and the process of articulating visually how technologies might be utilized in the future. In addition, the future workshop approach engages participants in discussions across two or more groups. This fosters a conducive climate for more divergent opinions to be voiced and in turn, overcome potential shortcomings associated with 'group think' in focus group [26, 27].

Blending FG with the future workshop approach allowed us to focus the group interview on the concept of 'context awareness' which we knew, from a single pilot interview with a doctoral student in the field of information systems, to be difficult to comprehend and envision for participants. Exercises adopted in the methodology facilitated participants in expressing their ideas about context awareness of a design scenario or a short description of a dream system in a concrete domain (using paper and pen) rather than in an abstract verbal form [28].

\subsection{Participants}

For the purpose of this study, a total 12 participants were recruited for two FGs, one in Denmark and one in China. All participants had more than 6 months of experience with smart phones. Each was compensated with a gift card for his/her participation. Six participants ( 3 male, 3 female) age between 22 and 31 were recruited in Denmark. All participants are Europeans and speak English.. Likewise, six participants (3 male, 3 female) age between 21 and 25 were recruited in China. All of them are ethnically Chinese and speak Mandarin. English is the language of communication for the FG in Denmark while participants conversed in Mandarin for the FG in China. 


\subsection{Moderators and Observers}

Both FGs in Denmark and China had one moderator and one observer. The moderator steered participants through the FG whereas the observer took charge of recording the procedure in audios, videos and photos. The Danish moderator was a male educator and researcher in the field of service design with more than 15 years of career experience. Similarly, the Chinese moderator was a male educator and researcher in the field of product and service design with more than 15 years of career experience.

\subsection{Procedure}

For participants in both FGs, it was first stressed that their participation did not constitute an evaluation of their academic performance. Each focus group comprised five progressive phases that lasted 2.5 hours including a short break. These five phases were: a warm-up phase, a brainstorming phase, a pros \& cons phase, a design phase and a validation phase. Transcripts from data gathered in the five phases were then divided into four main sections: Warm-up, Brain Strom, Pros \& Cons, Design and Validation.

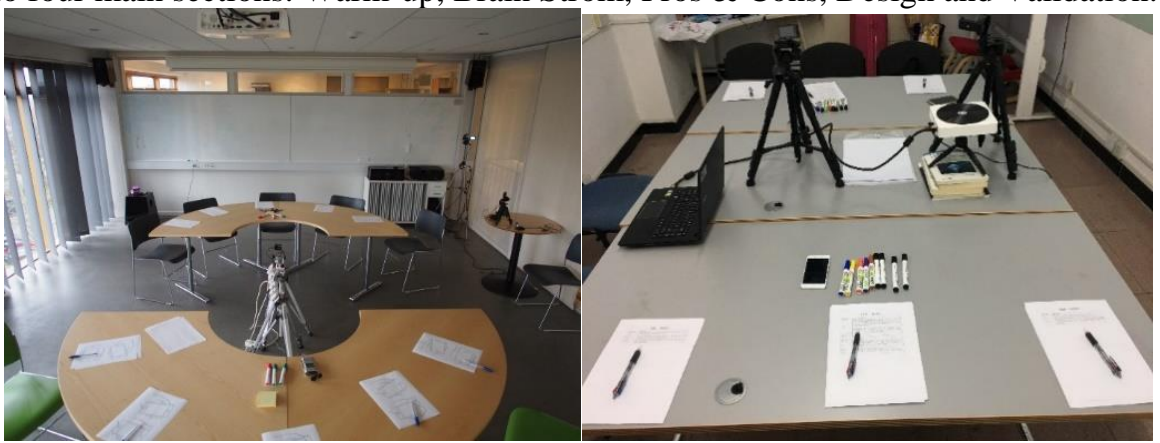

Fig. 1. Meeting room. Groups are placed at crescent tables, GoPro cameras record interactions in both groups. Secondary cameras record interactions and discussions between groups. Left side $=$ Focus group in Denmark, Right side=Focus group in China .

The focus groups were carried out in meeting/interview rooms at one university in Denmark and one in China. The setup included tables that separated the participants into two groups. GoPro cameras recorded each sub-group. Audio recorders captured group conversations. Further, two cameras were placed so as to capture both groups and the intergroup interaction. The layout of FGs in Denmark and China is depicted in Figure 1 below and the flow diagram for the FG procedure is illustrated in Figure 2.

\subsection{Material and Data Collection}

Printed template of mobile phone, pens and white board were distributed to participants for them to illustrate and sketch ideas. Data was collected in three formats: audio, video and photo. Four video cameras were deployed to record the FG from different angles. Two wide-angle GoPro cameras were placed at the front of each sub-group to record interactions within each group separately. Another two cameras were positioned at the 
two corners in the room to record the entire interaction process in the FGs from a holistic angle. Two audio recorders are fitted on the table of each FG to record the voices of participants. A photo camera was employed to record tangible outcomes of each FG, such as drawings, designs, sketches and presentations on whiteboard.

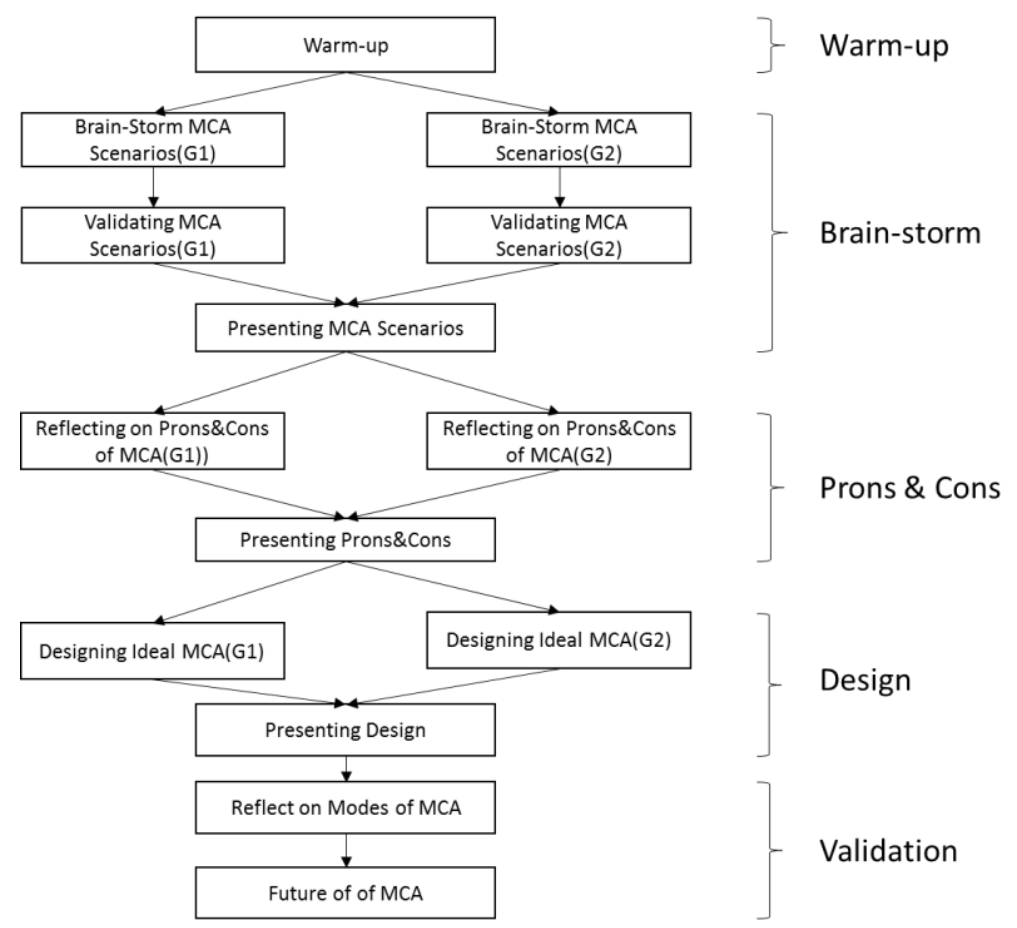

Fig.2. Chart of FG Procedure. MCA=Mobile Context-Awareness, WG=Within Group, BG=Between Group.

\subsection{Data Cleaning, Coding and Analysis}

Data in different formats are first matched with each other by timestamp and then transcribed into text for analysis. After cleaning the data, thematic analysis was adopted to encode and induce themes from the qualitative comments [29]. The objective of thematic analysis is to find a pattern in the random data that, at minimum, describes and organizes users' understanding of MCA and, at maximum, interprets the way that users structure MCA. The detailed procedure for thematic analysis is illustrated below:

- Isolating recurring patterns of interest with respect to context and context-aware applications: To make sure all researchers can understand and code the raw data, scripts from Chinese FG are translated into English. Three Western researchers generated a list of insights (i.e., codes + comments) based on the transcripts from the Danish FG. One researcher with a Chinese background listened in and supplemented the insights generated by the Western researchers. Next, Chinese researchers created a list of 
insights (i.e., codes + comments) on the basis of the Chinese FG. One of these was currently living in Denmark, and thus could compare across countries.

- Combining related patterns into themes: A researcher with a Chinese background and a researcher with Western background, supported by all (SKYPE call), compared the list of insights generated by both Chinese and Danish researchers in order to identify both commonalities and disparities in participants' attitude towards MCA across both cultural settings.

- Deriving excerpts from the FGs that correspond to these themes: A researcher went through the themes again and derived a potential framework for organizing the themes. Another researcher discussed with the first researcher about the structure and extract insights from the raw data to support the framework.

\section{Results}

It is well recognized that mobile applications offer an abundance of unique benefits by adjusting to user-specific circumstances and establishing a means to a connected lifestyle anytime and anywhere. Despite the potency of MCA, we still lack a fundamental understanding of how users evaluate MCA [19].

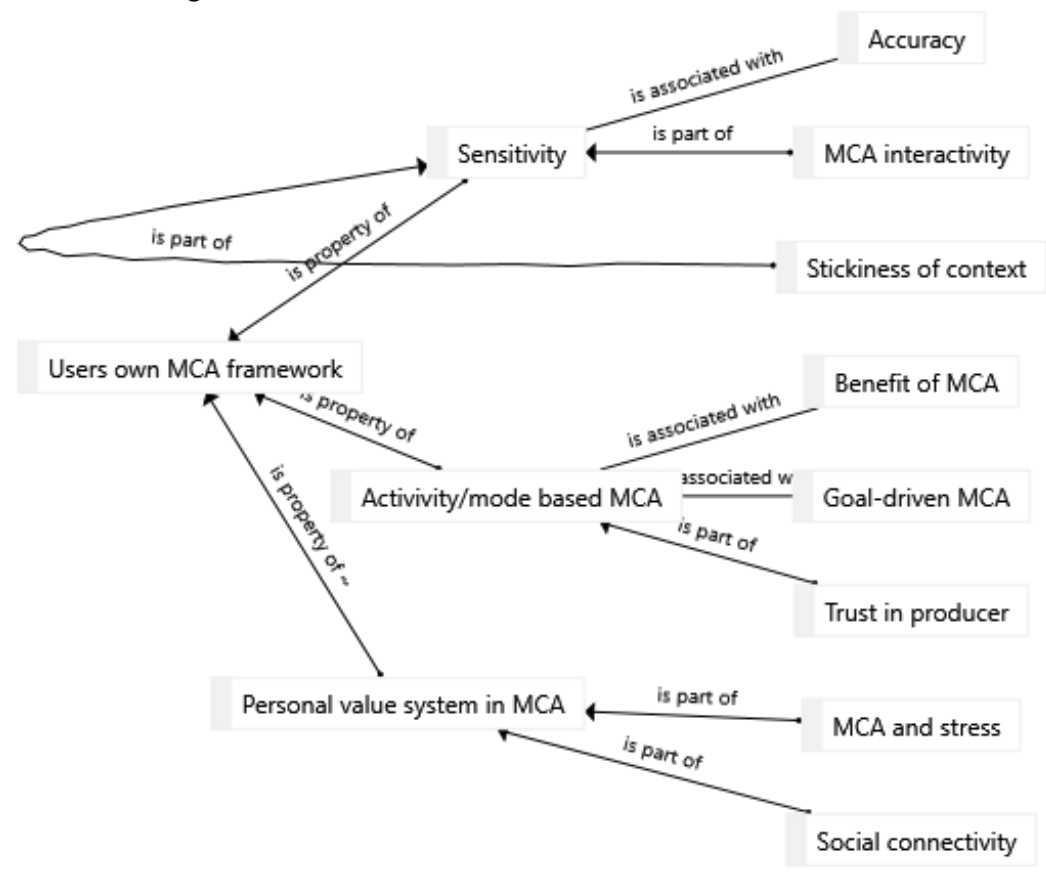

Fig.3. The categories of participants' views on MCA, and the interrelations.

Results from our thematic analysis reveals that participants can and do construct their own meaning of MCA, citing prominent examples of MCA as applied in everyday 
contexts. Second, participants' interpretation of MCA appears to be governed by their: (1) own personal value system; (2) sensitivity towards the degree of context-awareness for such systems, and; (3) their current activity in which they are engaged Each of these properties is associated with or constituted by other aspects of their understanding of MCA. This is exemplified through quotes from the focus groups below. Finally, participants are able to distinguish among the capabilities of mobile context-aware systems, the pros and cons of such systems as well as their views of how these systems could be designed from a user-centred angle (see Fig. 3).

\subsection{Participants' Own MCA framework}

One of the core findings in our study is how participants can and do construct their own meaning of MCA. They are able to supply examples of the use of MCA in everyday contexts. In Quote \#1 from the Danish FG during the Warm-up session, one of the participants explains what she thinks is an example of MCA.

MO Does anybody have sort of some impressions to share your examples about what context-awareness could be?

G2-CH For example, registering where you are could help you on vacation. Google Now has registered where I live and work automatically. And it does register some patterns where I'm going. That's an example from the mobile phone.

Quote \# 1 DK Warm-up Phase; MO = Moderator, G2-CH = Danish FG 2 Participant

G2-CH shares experiences from her own life. She knows about an application called Google Now that tracks its owner's location and displays it on the smartphone when it seems relevant, which G2-CH regards as an example of MCA. Another example is the participant in Quote \#2, from the Design phase in the Danish FG, who wants to share what she sees as the future of MCA.

G1-TR I have 3 different kind of scenarios.

The first one is that we (have to) get all these applications updated here in the AppStore. So either you can choose update it (all) or not. I would like to have the possibility of choosing or selecting new features in the update I like or I don't like. For instance, in Facebook, it says we have this new feature having favourite friends. But I dislike that feature. I still want to update the system without that feature. So it's kinda like individualizing the update.

And the other one is pre-setting your phone in different modes. I could make a mode called vacation mode. I pre-install it into (my phone). When I activate it, it knows that not to update my e-mail, or not give me alarm in the morning. And despite that when I deactivated it, I can activate it (anytime). That could be other modes, Whatever, likes sporting mode, maybe suggest some good news

And the last one is like digital business card that give each contact person ratings like those paid of loans, connects, and after that, we can arrange meetings.

Quote \# 2. DK Design Phase; G1-TR = Danish FG 1 Participant

G1-TR here, during the Design phase, sees herself as a co-designer, and has put forward several ideas for designing MCA. Some of these ideas appear to be centred on 
Western technologies such as App-store and Facebook, while others stem from activity/mode - vacation mode - to social connectivity - and business cards [see links in Fig. 3]. In a third example, Quote \#3, from the Brainstorm phase in the Chinese FG, a participant expresses his desire for a phone that is able to switch between the speaker for the human ear when he holds it closer to his head and the loudspeaker at the bottom of the phone whenever he holds it away from his head or it is covered by his clothing.

\section{G1-WW 通过手机的红外传感器来判断手机屏幕 Using infrared sensors to detect the dis- 与其它情境信息的距离, 比如使用微信 tance between screen of mobile phone 时通过与耳朵的距离来判断是否启动扬 and other contextual information. For ex- 声器模式, 以及被裤子覆盖的时候就自 ample, to switch on/off speaker mode ac- 动里屏，没有人会在屏暮被覆美的情况 cording to the distance between WeChat 动黑屏，没有人会在屏幕被覆盖的情况 (running on mobile phone) and ear; 下还使用手机 Switch off the screen when it is covered by trousers because no users will still use the phone when it's covered.}

Quote \# 3. CH Brainstorm Phase; G1-WW = Chinese FG 1 Participant

G1-WW is focused on the product itself and the sensitivity [see Fig. 3] of its MCA. He describes MCA in everyday Chinese context: the phone is referred to as the local technology WeChat when he mentions how the phone is covered by his trousers. He understands in his own framework how the phone is employing the speaker to play the voice talk or a blank screen, based on RFID sensor. Finally, in the fourth example, Quote \#4, a participant wonders if context-awareness is a nice-to-have function, feeling nostalgic about how lives were unaffected before MCA. She is afraid that once we have experienced the benefits of MCA, it might become a must-have function.

\section{G1-LC 以前没有智能手机，没有情境感知的时 We still lived good lives even without 候不是也过得很好? smart phones in the old days. \\ Quote \# 4. CH Design Phase; G1-LC = Chinese FG 1 Participant}

G1-LC relate MCA to her own life and personal values [see link in Fig. 3], reflecting on what it means to be human in the China at a time when everybody are looking more at their smart phones than at each other. In summary, participants' own understanding of MCA applications is shaped by their personal value systems, their sensitivity towards such applications, the current activity in which they are engaged and the cultural context they are living in. Participants' own frameworks for MCA are drawn from their experience with culturally-specific technologies (e.g., Facebook in the West and WeChat in China). Although users think that MCA is beneficial in improving mobile life, the benefit might be discounted due to failure of offering appropriate values to users.

\subsection{Components of Users' Own Understanding of MCA}

FG participants' comprehension of MCA appears to be grounded in their: (1) own personal value system; (2) sensitivity towards such applications, and; (3) current activity in which they are engaged. 
Personal Value System and MCA: MCA could be seen as a reflection of participants' personal value system. MCA devices can not only augment the quality of mobile life, but they can also enable users to realize socio-cultural values. MCA is hence deemed by participants to be a part of their socio-cultural value systems: MCA should fit their personal 'use case' and needs. In Quote \#5 from the Pros \& Cons phase in the Danish FG, two separate participants discuss the advantages and disadvantages of personal values afforded by MCA.

G1-TR I think context-awareness also helps you to recognize your personal values, so if you want to recycle, the phone can help you to have apps to collect food after the shop is closed. It's tailored to personal values.

G2-LI You can have this globalization on the same side of your personal values. You have more choices, but you are also provided with more directions to go. Maybe you can also get more pluralism

Quote \# 5. DK Pros \& Cons Phase. G1-TR, G2-Li = Danish FG 1 and 2 Participants

G1-TR appears to conclude that MCA can afford pluralism in personal values by permitting users to behave sustainably and at the same time, enjoy globalization (e.g., shopping and traveling). MCA reduce stress and increase social connectivity [see links in Fig. 3]. This is also the topic in Quote \#6 from the design phase in the Chinese FG where a participant alleges that system requirements could be proposed by humans.

\section{G2-CM 机器能做一切, 人只需要输入就好, 是 Machine can do everything and human

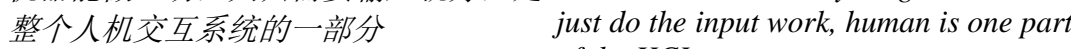 of the HCI system.}

Quote \# 6. CH Design Phase; G2-CM = Chinese FG 2 Participant

G2-CM sees the human user as part of a system where MCA is doing most of the work, and the human is merely stating her requirements and needs. Similarly, in Group 1 of the Chinese FG, a participant expresses her expectation of what MCA can do for humans: help them find friends.

G1-LC 在封闭环境中找到有共同爱好的人 MCA can help find people with common hobbies even in a closed society.

Quote \# 7. CH Pros \& Cons Phase; G1-LC = Chinese FG 1 Participant

G1-LC and G2-CM appears to see MCA as supportive of their personal value systems in which social connectivity and stress-free life [see links in Fig. 3] are important.

Participants' Sensitivity towards MCA: An important component of participants' own interpretation of MCA turned out to be sensitivity. We found that participants experienced the sensitivity of MCA, rather than just its presence or absence. They responded to the capability of context awareness. In Quote \#8 from the Warm-up phase in the Danish FG, everybody agrees on a lack of sensitivity of MCA in a situation where MCA interacts with the human user.

MO ... When you hold the phone like this, like vertically, then the text is running down vertically, but if you then turn it horizontally, then it responses to it, it flips and the text turns over in 90 degree to show it in horizontal view. So it's kind of sensitive to this kind of movement. So this is a very simple example of it. 
G1-TR But it is not always good. If you were leaning down, you want to turn it off because it is annoying to keep moving.

All Yeah(All)

Quote \# 8. DK Warm-up Phase; MO=Moderator, G1-TR = Danish FG 1 Participant

G1-TR employs negative wording to describe her own - and everybody's - UX of MCA interactivity: a lack of sensitivity. In Quote \#9 from the Brainstorm phase in the Danish FG, two participants gave another common example of poor sensitivity experiences caused by MCA's attempts at interactivity.

G2-El I choose the auto-connection of Wi-Fi. I like that when I'm in somewhere, my phone connects to Wi-Fi automatically. Because sometimes when the functionality was disabled, I forgot to do it. So I started to use it in roam, it uses my (cellular) data connection. In this case, it takes some money.

G2-El The bad side is that if I'm calling with Skype, the auto-connection will interrupt (my call). Every time it changes the connection, it has shit like this.

G2-LI Is that because the WIFI connection is not so good.

G2-El It's not, even the roam is so good. Because of the switch of connection, I hate that.

Quote \# 9. DK Brainstorm Phase; G1-EL. G2-LI = Danish FG 2 Participants

G2-EL hates when MCA switches from Wi-Fi to roaming since it costs her money and she either has to remember to take pre-emptive measures like disable the roaming or just get the bad feeling. In Quote \#10 from the Brainstorm phase in the Danish FG, participants lament whether reminders from Western transport companies (e.g., SAS = a regional airline) and information providers should incorporate MCA.

G1-TA Maybe just send reminders to you four hours before. Like don't forget that you have a flight in four hours. Or like actually send notification when this flight is late already, you just also don't have to worry about that. I mean it does show when you open it. That flight is not on time. Just give you notification that the flight is cancelled, delayed. That kind of notification works well.

G1-OR I don't know. I mean perhaps Google does that. Maybe SAS they don't have it.

G1-TA What do you mean?

G1-OR Actually I get the notification of my tickets some there, in e-mail? It's saved in my account

G1-TA Oh, no. I have like this application.

G1-OR But I mean they won't do that. Because they know Google is going to do that. So they don't want to get it

G1-TA But they sent me e-mail. They always sent me e-mail notification. So if I update e-mail notification I will be more aware of that. But it only shows in my e-mail. It's impossible if you want to see notifications about plane. Once I got SMS like your flight is delayed it would be better.

G1-TR But I think sometimes, for instances, you know the Danish train, the DSB, you buy one ticket .Then I have my phone number connected to its system and it will give me a text that my train is delayed. But what happens is a lot of time the text comes like already half an hour in the delay. It seems not working actually well, I hate they tell us people, I got the text that the train is late but they are not late very much. So apparently they sent out message to passengers that the train is just late for one minute. It sometimes causes more confusion. It actually wants to help you but it doesn't.

Quote \# 10. DK Brainstorm Phase; G1-TA, G1-OR, G1-TR = Danish FG 1 Participants 
G1-TR is upset when reminders are not timely according to her schedule and the MCA interactivity [see links in Fig. 3] (e.g., requirement to her about translating the reminder text that presents the untimely reminders). In Quote \#11 from the Design phase in the Danish FG, the same participants take the notion of MCA sensitivity to an extreme when they envision a system that can block them from going home to their families, compelling them to study instead.
G1-TR I think I have a lot of reasons to have this one. It's like having a Totalplanner in the way. It is basically an extension of you. Basically like open the iPod devices. You set up your goals, your timeline for the goals, your ethical background or something like that. So they are budgeted by rates. You put in your currencies. And then it creates an everyday-to-do-list for you to meet your goals by that time. It keeps like they are similarly. So it's like you put your time by the end of your semester. You have a number of, like 5, foreign languages. So basically, by controlling your lap- tops, told you now you have to study (2 hours, peers) like Java script, likes if you say I'm going abroad, I want go back to home land but it blocks you and says you have to study. So by the end of your semester, you fulfilled...
G1-TA That's a good one if it blocks
G1-TR Kind of very like time usage on your phone

Quote \# 11. DK Design Phase; G1-TA, G1-TR = Danish FG 1 Participants

G1-TA and G1-TR appear to design an insensitive system that can suppress their innate desire to go back to homeland through intimate interactions. In Quote \#12 from the Design phase in the Danish FG, a participant expresses her ideas for designing a socially sensitive human-like interactive MCA.

G2-LI We have a lot talk about interaction that could also be connected to the context of interaction, the social and physical, for example. If you don't have the screen, maybe it's strange in the public area just talk to the voice. Then you prefer smaller thing. Then if you are at home, you would like a bigger more. It is connected to the context, Other people, in the public area or private area, have less texting requirement, they just talk about all the time when they study and work. They just look at the screen, we just agreed, just goes like this from A to B. maybe it's nice to have talking instead of texting. But maybe if you get new knowledge in individual way unlike the previous one. Tomorrow, it's kind of different from just reading your text from the beginning to the end. At the same time, it should not talk too much, just imitate human being? There still to be something technology, not like too much, um, just pretending to be a human or to be helper

Quote \# 12. DK Design Phase; G2-LI = Danish FG 1 Participant

G2-LI is concerned with the sensitivity related to context of the MCA interactivity [see links in Fig.3], whether it is human voice or screen, at home or public, small or big. In Quote \#13, a participant from the Brainstorm phase in the Chinese FG also talks about the format of MCA interactivity, and mentions the fidelity of contextual information, like deploying VR to help people with visual impairment know the name and appearance of the buildings nearby. 
G2-CM 最后个是 VR 就是能告诉你你旁边的建筑 The last case is VR, it can tell you the name of the building besides you and then 物的名字, 这样可以帮着路痴的人知道怎 it can guide the road-blind people to the 么走 right way.

Quote \# 13. CH Brainstorm Phase; G2-CM = Chinese FG 2 Participant

G2-CM is concerned with the sensitivity of the MCA in a VR environment for specific user groups like the blind. This is also associated with accuracy of the MCA [see links in Fig. 3], how precise it is. In Quote \#14 from the Brainstorm phase in the Chinese FG, a participant talks about how MCA should offer emotional songs according to personal use history and how MCA scenarios transform users' mobile lives.

G2-ZY 能根据不同地方听的歌的类型来给出建 Can give suggestions according to the types of songs in different places. For ex议, 比如每次去明光楼都放欢快的歌, ample, I tend to listen to brisk songs every 那么以后就在明光楼附近时就提供欢快 time I'm in Mingguang Building, then it 的歌 should offer me cheerful songs automatically every time I'm around Mingguang Building

Quote \# 14. CH Brainstorm Phase; G2-ZY = Chinese FG 2 Participant

G2-ZY requires MCA to be very accurate across geographic and emotional contexts. Furthermore, she is alluding to the stickiness of context [see links in Fig. 3]. To her, the Mingguang building is a recurring context that embodies emotional and maybe even abstract, metaphorical value. Finally, Quote \#15 from the Pros \& Cons phase is just plainly underscoring the need for accurate and sensitive MCA.

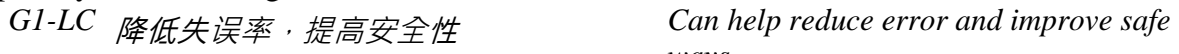
ways

Quote \# 15. CH Pros \& Cons Phase; G1-LC = Chinese FG 1 Participant

G1-LC asks for MCA that can improve the safety of accomplishing tasks. In sum, sensitivity is a major component of the participants' own MCA framework, and it relates to the accuracy, interactivity and stickiness of MCA.

Participants' Appreciation of Activity Accommodated by MCA: A third component of participants' own MCA framework is the activity accommodated by MCA. This occurs when users experience the device as the source of MCA and when they experience select applications as the sources of MCA during activities. It is associated with benefits of MCA, goal-driven MCA and part of it is to have trust in the provider of MCA. In Quote \#16 from the Brainstorm phase in the Danish FG, a participant discusses about the issue of Wi-Fi auto connection produced by a local Danish university of the participants (i.e., CBS), versus roaming, in the context of video telephony with a Western application (i.e., Skype).

G2-El The last one is a very simple function of smart phone. It's the auto connection to the WIFI. For example, when I came here, the phone recognizes the WIFI of CBS and connect. It's really very good because I can save some money. The bad side is that if I'm doing a Skype call, the connection goes, the auto connections switches from 
my normal connection to WIFI and the Skype call is done at the time, and the time to recall and lose time by that. So it's a function of the phone that it is able to understand the time to switch. The phone has sense to know when the Skype call is on-going and can't be interrupted, it is not going to switch now.

Quote \# 16. DK Brainstorm Phase; G2-EL = Danish FG 2 Participant

G2-EL does not completely trust the provider [see links in Fig. 3] of her Wi-Fi internet connection, and therefore her telephony MCA experience varies from good to bad, depending on which mode her connection is in. In Quote \# 17 from the Brainstorm phase in the Danish FG, two participants, being in Copenhagen that brands itself as a city for bicycles, advocated a 'Bike mode' that allows users to dictate the MCA experience by controlling which mode the MCA device or application is in.

G1-TR I don't know whether there are already existing phone holder for your bicycle. I haven't tried it. I don't think it can solve your entry problems. But I definitely think it's important to think about what settings the phone is in. The phone might interfere with whatever you were doing with the smartness functionality, so it has to think about the contexts of your surroundings, too.

G1-TA But it could have like a button, you know, pull down from here, you have to choose different settings, like a Bike Mode. Exactly just like you said, the screen will be on all the time. That maybe can work likes, whether or not it's going to detect that you're on the bike specifically, because I don't know how would it to do that? But it's like other group told us, the user can just push this button manually, it has everything to do for being on the bike.

Quote \# 17. DK Brainstorm Phase; G1-TR, G1-TA = Danish FG 1 Participants

G1-TR and G1-TA also touches on how much trust you can place into the activitybased MCA, and decides that not even when the user decides on the mode, the MCA device can be trusted to provide a consistent satisfactory experience. In Quote \#18 from the Brainstorm phase in the Chinese FG, a participant talks about local applications of WeChat and Weibo that monitors user behaviour, but do not deliver any benefits arising from this MCA.

G1-LC 有时在监测我们的行为, 但没有转化成 Sometimes the phone is supervising our 有用的信息担供给们 有用的信息提供给我们, 比如购买行为 to transform the data into useful infor, 微博微信上的点赞行为, 翻译成文字 mation for us. For example, the purchase

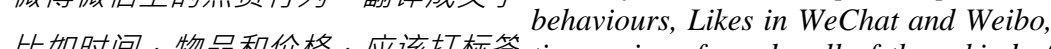
, 比如时间, 物品和价格, 应该打标签 time, price of goods, all of these kind of , 汇总一下, 方便做总结任formation should be tagged and integrated for making summary (to come up with valuable information),

Quote \# 18. CH Brainstorm Phase; G1-LC = Chinese FG 1 Participant

G1-LC points out that it is not enough to perform automated analysis and summarize online purchase behaviours. Rather, as she sees it, any data-mining measures undertaken by MCA should deliver concise benefits to users [see links in Fig. 3]. In Quote \#19 from the Brainstorm phase in the Chinese FG, a participant outlines the idea that 
MCA, much like the auto-completion feature for drawing, should be able to auto-complete a drawing that users begins to draw. Being in China, this could mean auto-completing the writing of Chinese characters based on initial strokes.

\section{$G 1-L C$ 知道我要画什么, 自动就把画给画出来 \\ , 象微软的文字联想一样}

Functionality of Drawing Association like text association that can predict my intention and finish the rest of my drawing automatically

Quote \# 19. CH Brainstorm Phase; G1-LC = Chinese FG 1 Participant

G1-LC identifies a possible benefit by suggesting that the MCA should be driven by the same goals as its user, a goal-driven MCA [see links in Fig. 3]. In Quote \#20 from the Pros \& Cons phase in the Chinese FG, a participant touches on the nature of human activities today.

\section{G1-LC 导致时间碎片化, 抢占了时间年洋e is fragmented and occupied \\ Quote \# 20. CH Pros \& Cons Phase. G1-LC = Chinese FG 1 Participant}

G1-LC is here alluding to the all-important requirement of MCA to support the user in managing fragmented and occupied time.

In summary, the third component of the users' own MCA framework is activity based MCA, which is associated with benefits of MCA and goal-driven MCA and partly influenced by their trust in the provider(s) of MCA.

Participants' Understanding of MCA in Different Phases of the Design Process: In this study, we have opted to concentrate on users' experience of MCA in various phases in the overall design process. We observe that there is the UX of MCA differs across distinct design process phases. By blending the user-centred taxonomy of MCA (Fig. 3) and the design process that we espouse in the FG (Fig. 2), we can arrive at a taxonomy/paradigm (fig. 4, see also [30]) that visualizes the pattern of UX of MCA through the distribution of Quotes \#1-20, both for the Danish and Chinese FGs.

Fig. 4 reveals that the participants reflect on their daily activities during the Warmup and Brainstorm phases, and what they value during the Pros \& Cons phase. Furthermore, they thought about the MCA device itself and its related sensitivity during the Warm-up phase (to supply both good and bad examples of MCA) and during the Design/Validation phase (to put forth ideal solutions).

\begin{tabular}{|c|c|c|c|c|c|c|c|c|}
\hline \multirow{2}{*}{$\begin{array}{c}\text { Location } \\
\text { Phase }\end{array}$} & \multicolumn{4}{|c|}{ Copenhagen } & \multicolumn{4}{|c|}{ Beijing } \\
\hline & $\begin{array}{l}\text { Warm- } \\
\text { up }\end{array}$ & $\begin{array}{l}\text { Brain- } \\
\text { storm }\end{array}$ & $\begin{array}{c}\text { Pros \& } \\
\text { Cons }\end{array}$ & Design & $\begin{array}{c}\text { Warm } \\
\text {-up }\end{array}$ & $\begin{array}{l}\text { Brain- } \\
\text { storm }\end{array}$ & $\begin{array}{c}\text { Pros \& } \\
\text { Cons }\end{array}$ & $\begin{array}{l}\text { De- } \\
\text { sign }\end{array}$ \\
\hline $\begin{array}{l}\text { Users' Own Frame- } \\
\text { work }\end{array}$ & $\# 1$ & - & - & \#2 & - & \#3 & - & \#4 \\
\hline Activity/Mode-based & - & $\# 16,17$ & - & - & - & $\# 18,19$ & \#20 & - \\
\hline Personal Value System & - & - & \#5 & - & - & - & \#7 & \#6 \\
\hline Sensitivity & \#8 & $\# 9,10$ & - & $\begin{array}{c}\# 11, \\
12\end{array}$ & - & $\# 13,14$ & \#15 & - \\
\hline
\end{tabular}

Fig. 4. Participants' Understanding of MCA across FG Phases in two Locations 


\section{Discussion}

This paper demonstrates that UX of MCA, as uncovered through FGs and future workshops in Denmark and China, are not simple outcomes of technological or user attributes. Rather, it embodies dynamic and situation-specific experiences that are shaped by cultural characteristics and design process elements.

\subsection{MCA is a Function of Mobile Life}

It is evident that users possess their own meaning of MCA because they can envision and supply examples on the application of MCA in daily contexts. In contrast to contemporary MCA studies that conceive context awareness as a property of the device [14], this study attests to the necessity of treating context-awareness as a HCI function, which can be assimilated into users' daily mobile lives. The development of contextawareness applications should hence extend beyond conceptual and technical boundaries to take into account users' attitude, intention and activity [8]. While mobile applications facilitate a connected lifestyle, MCA has the potency to render the connected lifestyle more accommodating of and tailored to users' physical, psychological and social characteristics. In turn, the middleware of MCA should be capable of capturing, interpreting and inferring contextual information pertinent to users' lifestyle.

\subsection{Users Construct MCA with Three Dimensions}

A growing body of research on consumers' value perceptions in context of mobile applications alleged that the value offered by these applications is multidimensional, which might entail functional, social, emotional, epistemic, political, moral, spiritual and conditional value. Nevertheless, there is still little consensus on how to best conceptualize value perceptions in that the construction of value tend to vary across contexts [19]. The current study proffers insights into the structure and dimensions of MCA from users' standpoint.

Users' appreciation of MCA appears to be founded on their: (1) own personal value system; (2) sensitivity towards such applications, and; (3) current activity in which they are engaged. While contemporary MCA studies tend to construe context-awareness from the conceptual, technological and infrastructural angles, this study discovers that high quality MCA applications depends on three discrete elements: sensitivity, human activity and value systems. Future research should thus investigate users' interactions with MCA in their daily lives. Any conceptual or technological exploration of MCA must not discount the sensitivity, activity and value systems of humans so as offer an enhanced UX for mobile applications.

\subsection{Cross Cultural Issues in MCA}

In our study, both FGs in Denmark and China were fearful that intelligent mobile applications might threaten human evolution by reducing the requirement for cognitive 
processing. Consequently, active MCA for capturing and reasoning contextual information, and passive MCA for executing contextual inferences, constitute universal preferences across both cultural groups. This study also identified several potential cultural differences. Denmark participants believed that the design of MCA might be culture-dependent. Integrating multiple functionalities (e.g., messaging, social networking and payment) into a single application, while commonly practised in China, is rare in the West, which may reflect regional differences in practices in reasoning and thinking $[10,11]$. This implies that the contextual information embedded within one mobile application, and the usage of this contextual information, might be much more sophisticated in China, a design complication that is of greater concern to Westerners [13].

Our participants' own frameworks for MCA were grounded in their experience with culturally-unique technologies (e.g., Facebook in the West and WeChat in China). Closer scrutiny of our empirical instances (see Quotes \#1-20), shows that users' MCA scenarios rely on local lives and technologies with major distinctions: Facebook, Google, SAS, Netflix, Total-planner, and Bike, are mentioned by Danish participants, while Wechat, Weibo, Huawei, Smartisan, music, B2C and drawing characters are cited by their Chinese counterparts. The UX of MCA appears as culturally oriented towards the way that MCA is experienced and its variation in UX across design phases, i.e., cultural aspects of UX of MCA appears to be both dynamic and situation specific [11].

\section{Limitations and Future Work}

The current study has a number of caveats. Regarding the methodology, as an exploratory study aimed at deciphering how users draw meaning from MCA, FG and future workshops were deployed in this study. Though the methodology is invaluable in helping to get insights on UX, it is insufficient in delivering a structured and validated framework. More structured studies are required to validate our empirical findings. Regarding the user sample, twelve mobile device users participated in this study and their understanding of MCA was covered in depth. The UX of MCA system rely very much on the usage purpose and user profiles such as physical, psychological and social characteristics. A more comprehensive research involving a greater number of participants can aid in verifying the generalizability of our empirical findings. Finally, an ecological issue emerged, as this study was conducted in a FG room by prompting participants to disclose their opinions of MCA in accordance with their previous experiences. It is probable that salient factors might have been excluded from this study unintentionally or deliberately omitted by participants out of privacy considerations or time constraints. Future research into MCA can perhaps turn to field studies as a means of refining our empirical findings. To overcome the limitations of this study, we will: (1) replicate the findings in the field by performing contextual inquiries in the form of daily diaries and usage logs; (2) develop a structured survey questionnaire to measure UXs with MCA.

\section{Acknowledgement}

This study is part of the project Mobile context-aware cross-cultural applications (MOCCA) funded by Marie Skłodowska-Curie Action, grant number 708122. 


\section{References}

1. Statista, https://www.statista.com/statistics/330695/number-of-smartphone-usersworldwide/

2. Baldauf, M., Dustdar, S., \& Rosenberg, F.. A survey on context-aware systems. International Journal of Ad Hoc and Ubiquitous Computing, 2(4), 263-277 (2007)

3. Cheverst, K., Davies, N., Mitchell, K., Friday, A., \& Efstratiou, C.. Developing a context-aware electronic tourist guide: some issues and experiences. In Proceedings of the SIGCHI conference on Human Factors in Computing Systems. 1724(2000)

4. Sarjakoski, L. T., \& Nivala, A. M.. Adaptation to context-a way to improve the usability of mobile maps. In Map-based mobile services (pp. 107-123). Springer Berlin Heidelberg(2005).

5. Kaasinen, E.. User needs for location-aware mobile services. Personal and ubiquitous computing, 7(1), 70-79 (2003).

6. Dey, A.K., Häkkilä, J.: Context-Awareness and Mobile Devices. User interface design and evaluation for mobile technology. 1, 205-217 (2008).

7. Hong, J.Y., Suh, E. H., Kim, S. J.: Context-aware systems: A literature review and classification. Expert Systems with Applications. 36(4), 8509-8522 (2009).

8. Barkhuus, L., Dey, A.: Is Context-Aware Computing Taking Control away from the User? Three Levels of Interactivity Examined. In: UbiComp 2003: Ubiquitous Computing, pp. 149-156.Springer, Heidelberg (2003).

9. Samie, M.A.: Human centric situational awareness. Master Thesis, The American University in Cairo (2014).

10. Friedman, R., Liu,W., Chi, S.C. S., et al.: Cross-cultural management and bicultural identity integration: When does experience abroad lead to appropriate cultural switching?. Social Science Electronic Publishing. 36 (1),130-139 (2012).

11. Hong, Y. Y.: A dynamic constructivist approach to culture: Moving from describing culture to explaining culture.3-23 (2009).

12. Hong, Y. Y., Mallorie. L.A.M.: A dynamic constructivist approach to culture: Lessons learned from personality psychology. Journal of Research in Personality.38(1):59-67 (2004).

13. Frandsen-Thorlacius, O., Hornbæk, K., Hertzumm, M., et al. Non-universal usability? a survey of how usability is understood by Chinese and Danish users. In: Proceedings of the SIGCHI Conference on Human Factors in Computing Systems. 41-50 (2009).

14. Schilit, B. N., Theimer, M. M.: Disseminating active map information to mobile hosts. IEEE Network. 8(5),22-32 (1994).

15. Abowd, G. D., Dey, A.K., Brown, P.J., et al.: Towards a better understanding of context and context-awareness. Huc '99 Proceedings of International Symposium on Handheld \& Ubiquitous Computing. 1707,304-307 (1999).

16. Schmidt, A.: Implicit human computer interaction through context. Personal and Ubiquitous Computing. 4(2),191-199 (2000).

17. Rötting, M, Zander, T., Trösterer, S., et al. Implicit Interaction in Multimodal Human-Machine Systems. Industrial Engineering and Ergonomics. Springer Berlin Heidelberg. 523-536 (2009). 
18. Leonard-Barton, D., \& Sinha, D. K Developer-user interaction and user satisfaction in internal technology transfer. Academy of Management Journal, 36(5), 1125-1139 (1993)

19. Gummerus, J., \& Pihlström, M.. Context and mobile services' value-in-use. Journal of Retailing and Consumer Services, 18(6), 521-533 (2011)

20. Gallego, D., Woerndl, W., \& Huecas, G.. Evaluating the impact of proactivity in the user experience of a context-aware restaurant recommender for Android smartphones. Journal of Systems Architecture,59(9), 748-758(2013).

21. Merton, R. K.: The focused interview and focus groups: continuities and discontinuities. Public Opinion Quarterly. 51, 550-556 (1987).

22. Lunt, P. \& Livingstone, S.: Rethinking the focus group in media and communications research. Journal of Communication. 46(2), 79-98 (1996).

23. Morgan, D. L.: Focus Groups. Annual Review of Sociology.22,129-152 (1996).

24. Jungk, R. \& Müllert, N.: Future workshops: How to Create Desirable Futures. London, England, Institute for Social Inventions. (1987).

25. Ehn, P. and Sjögren, D.: From System Description to Scripts for Action. In Greenbaum, J. and Kyng, M. (eds.). Design at Work: Cooperative Design of Computer Systems. Lawrence Erlbaum Associates, Hillsdale, NJ, pp. 241-268 (1991).

26. Kensing, F. \& Madsen, K. H.: Generating Visions: Future Workshops and Metaphorical Design. In Greenbaum, J. \& Kyng, M. (eds), Design at Work: Cooperative Design of Computer Systems. New Jersey, USA: Lawrence Earlbaum Associates. (1991).

27. Carey, M. A., \& Smith, M. W.: Capturing the group effect in focus groups: A special concern in analysis. Qualitative Health Research. 4(1), 123-127 (1994).

28. Bødker, S.: Scenarios in user-centred design: Setting the stage for reflection and action. Proceedings of the 32nd Hawaii International Conference on System Sciences, Maui, HI.(1999).

29. Boyatzis, R. E.. Transforming qualitative information: Thematic analysis and code development. (1998)

30. Spradley, J. (1979). The ethnographic interview. New York: Holt, Rinehart and Winston. 